\title{
Técnicas de caracterização de porosidade para o controle de misturas de concreto permeável
}

\author{
R. J. Mikami ${ }^{1,2 *}$, E. Pereira ${ }^{1,2}$ \\ *Autor de Correspondência: rjmikami@uepg.br \\ ${ }^{1}$ Programa de Pós-Graduação em Engenharia e Ciência de Materiais, Universidade Estadual de Ponta Grossa, Ponta \\ Grossa, Brasil \\ ${ }^{2}$ Departamento de Engenharia Civil, Universidade Estadual de Ponta Grossa, Ponta Grossa, Brasil
}

\begin{abstract}
RESUMO
A porosidade em concretos permeáveis é um parâmetro imprescindível no controle de qualidade, influenciando a durabilidade e eficiência de pavimento drenantes. Este trabalho teve como objetivo comparar técnicas de caracterização da porosidade de misturas de concreto e avaliar a influência no controle das propriedades mecânicas e hidráulicas. Foram produzidas duas misturas de concreto permeável compactadas com diferentes energias para produzir porosidades distintas. Realizou-se a caracterização da porosidade, resistência e condutividade hidráulica. Com os resultados comprovou-se a relação entre a porosidade no estado fresco e endurecido. Foi possível construir modelos de previsão de condutividade hidráulica e resistência utilizando-se a porosidade como preditor. Os resultados demonstram a importância do controle da porosidade, uma vez que influencia propriedades essenciais para pavimentos drenantes.
\end{abstract}

Palavras-chave: pavimento permeável; porosidade; estado fresco; resistência; permeabilidade

\section{ABSTRACT}


Porosity in pervious concretes is an essential parameter in quality control, influencing the durability and efficiency of draining pavements. This study aimed to compare techniques for characterizing the porosity of concrete mixtures and to evaluate the influence on the control of mechanical and hydraulic properties. Two pervious concrete mixtures compacted with different energies were produced to produce different porosities. The characterization of porosity, strength and hydraulic conductivity was carried out. The results proved the relationship between porosity in the fresh and hardened state. It was possible to build models for predicting hydraulic conductivity and strength using porosity as a predictor. The results demonstrate the importance of controlling porosity, since it influences essential properties for draining floors.

Keywords: pervious pavement; porosity; fresh state; strength; permeability

\section{RESUMEN}

La porosidad en el hormigón permeable es un parámetro esencial en el control de calidad, que influye en la durabilidad y eficiencia de los pavimentos drenantes. Este trabajo tuvo como objetivo comparar técnicas para caracterizar la porosidad de mezclas de hormigón y evaluar la influencia en el control de propiedades mecánicas e hidráulicas. Se produjeron dos mezclas de hormigón permeable compactado con diferentes energías para producir diferentes porosidades. Se realizó la caracterización de porosidad, resistencia y conductividad hidráulica. Los resultados demostraron la relación entre la porosidad en estado fresco y endurecido. Fue posible construir modelos para predecir la conductividad hidráulica y la resistencia utilizando la porosidad como predictor. Los resultados demuestran la importancia de controlar la porosidad, ya que influye en propiedades esenciales para el drenaje de suelos.

Palabras clave: pavimento permeable; porosidad; estado fresco; resistencia; permeabilidad

\section{INTRODUÇÃO}

O concreto permeável é um material que tem se destacado como uma das alternativas para a mitigação de inundações urbanas (Xie et al., 2018). Com uma capacidade de infiltração elevada, o material pode ser utilizado em pavimentos permeáveis com o intuito de reduzir o escoamento superficial das águas pluviais. Essas estruturas têm se tornado atrativas por sua natureza multifuncional, funcionando como pavimentos para o tráfego de veículos e, simultaneamente, como componentes do sistema de drenagem urbana (Chandrappa e Biligiri, 2016). No entanto, o desempenho e durabilidade dos pavimentos permeáveis depende diretamente das suas propriedades mecânicas e hidráulicas.

Para garantir a qualidade de um pavimento drenante, o concreto permeável deve ser dosado para atingir uma resistência mecânica suficiente para resistir aos esforços oriundos do tráfego de veículos. Ao mesmo tempo, o material ainda deve manter um coeficiente de permeabilidade suficiente para garantir a rápida infiltração da água no pavimento. Porém, estas propriedades são inversamente proporcionais, sendo essa a principal dificuldade no processo de dosagem do material (Bonicelli et al., 2013; Gaedicke et al., 2015).

A porosidade é um dos parâmetros chave na dosagem misturas de concreto permeável, afetando a maioria das suas propriedades. Os concretos permeáveis usualmente são projetados com porosidades variando entre 15 e 30\% (Zhong e Wille, 2015; Ibrahim et al., 2014). Dentro dessa faixa ampla, o volume de poros deve ser ajustado conforme a resistência mecânica e condutividade hidráulica desejadas. Dessa forma, o controle da porosidade é uma etapa fundamental para 
assegurar a qualidade do concreto permeável e garantir a sua funcionalidade dentro de um pavimento.

Além disso, é necessário avaliar como as características das misturas de concreto permeável irão impactar a durabilidade dos pavimentos drenantes. Quando expostos às intempéries, os pavimentos permeáveis são suscetíveis ao efeito de colmatação dos poros, causado pelo acúmulo de sedimentos. A colmatação reduz significativamente a permeabilidade do material, prejudicando a sua durabilidade (Rao et al., 2020). O tamponamento dos vazios do concreto permeável depende não apenas da porosidade global do material, mas também de características como dimensão, forma e conectividade dos poros (Kia et al., 2017).

Portanto, ainda que seja uma propriedade importante, não há uma padronização na caracterização da porosidade dos concretos permeáveis. Embora muitas vezes não seja realizada nenhuma distinção, a porosidade do concreto permeável pode ser subdividida em três grupos, conforme a Figura 1.

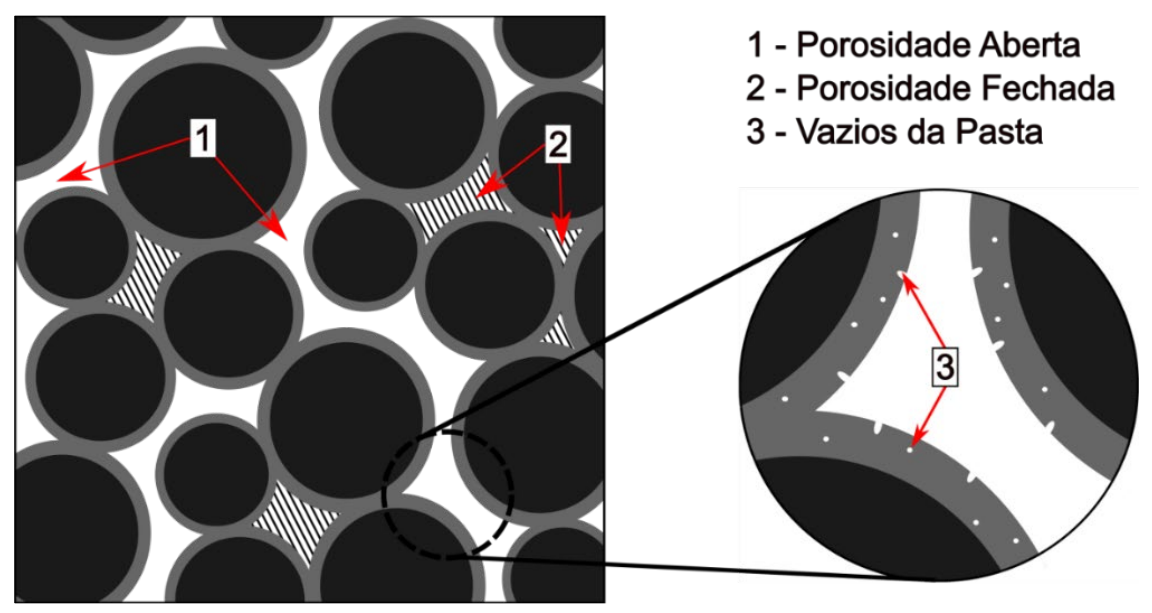

Figura 1. Classificação dos poros presentes no concreto permeável

Conforme ilustrado na Figura 1, a porosidade aberta consiste nos poros interligados capazes de conduzir a água pelo interior do material (Joshaghani, 2015). A porosidade fechada, por outro lado, é formada por poros isolados e que não contribuem para a condutividade hidráulica do concreto (Torres e Hu, 2016). A soma dessas duas porosidades é denominada de porosidade integranular do concreto permeável. A matriz cimentícia do concreto permeável também apresenta uma porosidade, denominada neste estudo de vazios da pasta de cimento (Mehta e Monteiro, 2008). A soma das três parcelas de poros usualmente é denominada de porosidade total.

A determinação da porosidade pode ser realizada no estado fresco, conforme a ASTM C1688M (ASTM, 2014), e no estado endurecido, conforme a ASTM C1754M (ASTM, 2012). Além dessas duas metodologias, a porosidade aberta do concreto permeável também pode ser caracterizada por um método proposto por Deo e Neitalath (2010). O resultado obtido por cada uma dessas metodologias será diferente, o que pode ser atribuído aos tipos de poros considerados em cada técnica de caracterização. Para compreender como as propriedades do concreto permeável são afetadas pela porosidade, é fundamental distinguir os diferentes tipos de vazios e conhecer as técnicas de caracterização adequadas. Dessa forma, o objetivo deste estudo foi realizar uma análise comparativa entre as metodologias de caracterização da porosidade e estabelecer uma relação entre estas e as propriedades mecânicas e hidráulicas do concreto permeável. 


\section{METODOLOGIA}

\subsection{Materiais e Dosagem da Mistura de Concreto Permeável}

Na produção das misturas de concreto permeável foi utilizado o cimento Portland CP II F-32 e brita 0 . O agregado graúdo apresentou uma distribuição granulométrica conforme a classe ASTM $\mathrm{n}^{\mathrm{o}} 89$, com massa específica de $3,05 \mathrm{~g} / \mathrm{cm}^{3}$ e massa unitária no estado compactado de $1,67 \mathrm{~g} / \mathrm{cm}^{3}$. Foram desenvolvidos dois traços de concreto permeável, com o objetivo de estabelecer porosidades de 20 e $30 \%$ para um nível de compactação inicial. Para alcançar essas porosidades foram utilizadas relações agregado/cimento $(\mathrm{g} / \mathrm{c})$ de 3,10 e 4,68, respectivamente. Todos os concretos foram elaborados com relação água/cimento $(\mathrm{a} / \mathrm{c})$ de 0,28 .

Cada mistura de concreto permeável foi compactada utilizando diferentes métodos de compactação para avaliar o impacto na porosidade. A compactação foi realizada utilizando-se um soquete Proctor com massa de $2,5 \mathrm{~kg}$ e altura de queda de $305 \mathrm{~mm}$. O esforço de compactação foi variado ajustando-se o número de camadas de adensamento e o número de golpes aplicado por camada, conforme descrito na Tabela 1.

Tabela 1. Métodos de compactação empregados na produção das misturas de concreto permeável

\begin{tabular}{|c|c|c|}
\hline Método de Compactação & $\mathbf{N}^{\mathbf{0}}$ Camadas & $\mathbf{N}^{\mathbf{0}}$ Golpes \\
\hline A & 2 & 10 \\
\hline B & 3 & 6 \\
\hline C & 2 & 15 \\
\hline D & 3 & 10 \\
\hline E & 2 & 20 \\
\hline F & 3 & 14 \\
\hline
\end{tabular}

Foi produzido um total de 12 misturas de concreto (2 relações g/c e 6 métodos de compactação), sendo moldadas 8 amostras cilíndricas com $10 \mathrm{~cm}$ de diâmetro e $20 \mathrm{~cm}$ de altura, totalizando 96 corpos de prova. Das 8 repetições, 4 foram destinadas ao teste de resistência à compressão axial e as demais utilizadas na determinação da porosidade e coeficiente de permeabilidade.

\subsection{Técnicas de Caracterização da Porosidade}

Neste estudo a porosidade do concreto permeável foi determinada por quatro metodologias distintas. A porosidade no estado fresco foi determinada seguindo o método descrito na ASTM C1688M (ASTM, 2014). No estado endurecido a propriedade foi determinada pelo procedimento descrito na ASTM C1754M (ASTM, 2012), baseado na pesagem hidrostática das amostras de concreto. A porosidade aberta também foi determinada por uma metodologia adaptada do estudo de Deo e Neithalath (2010). Neste método as amostras foram mantidas em estufa a uma temperatura de $100^{\circ} \mathrm{C}$ até constância de massa $\left(\mathrm{m}_{\text {est }}\right)$. Na sequência, foi realizada a vedação das laterais e base da amostra de concreto permeável com o auxílio de uma membrana plástica. Para determinar a porosidade, água é adicionada ao concreto permeável até que a amostra seja completamente saturada $\left(\mathrm{m}_{\mathrm{sat}}\right)$. $\mathrm{O}$ volume de água adicionado à amostra corresponde ao volume de poros abertos no concreto permeável, conforme determinado na equação 1 .

$$
\mathrm{Pa}=\frac{\mathrm{m}_{\mathrm{sat}}-\mathrm{m}_{\mathrm{est}}}{\mathrm{V}}
$$

Sendo $\mathrm{Pa}$ a porosidade aberta $(\%), \mathrm{m}_{\text {sat }}$ a massa da amostra preenchida com água $(\mathrm{g}), \mathrm{m}_{\text {est }}$ a massa da amostra seca em estufa (g) e V o volume da amostra $\left(\mathrm{cm}^{3}\right)$. 
Neste estudo foi proposta uma metodologia para caracterizar a porosidade intergranular. Para isto, foi realizado um ensaio para determinar a massa específica aparente do concreto permeável. Esse teste consistiu em fragmentar uma amostra de concreto permeável em grãos (compostos por agregado e pasta de cimento) e submetê-los à pesagem hidrostática, pelo mesmo procedimento utilizado na determinação da massa específica de agregados graúdos, conforme a NBR NM 53 (ABNT, 2009). Na sequência, a porosidade intergranular (Pi) pode ser estimada por meio de uma relação entre a densidade aparente do fragmento de concreto $\left(\mathrm{d}_{\mathrm{ap}}\right)$ e da massa específica do concreto (Mesp), conforme a equação 2.

$$
\mathrm{Pi}=\frac{\mathrm{d}_{\mathrm{ap}}-\mathrm{M}_{\mathrm{esp}}}{\mathrm{d}_{\mathrm{ap}}}
$$

Em que Pi é a porosidade intergranular no estado endurecido (\%), $d_{a p}$ é a massa específica aparente do fragmento de concreto $\left(\mathrm{g} / \mathrm{cm}^{3}\right)$ e $\mathrm{M}_{\text {esp }}$ é a massa específica do concreto permeável $\left(\mathrm{g} / \mathrm{cm}^{3}\right)$.

\subsection{Condutividade Hidráulica}

A condutividade hidráulica do concreto permeável foi determinada utilizando-se um permeâmetro de carga variável, conforme recomendado pela ACI 522R-10 (ACI, 2010). Para o desenvolvimento deste estudo foi elaborado um permeâmetro com capacidade de carga hidráulica de $50 \mathrm{~cm}$, ilustrado na Figura 2.

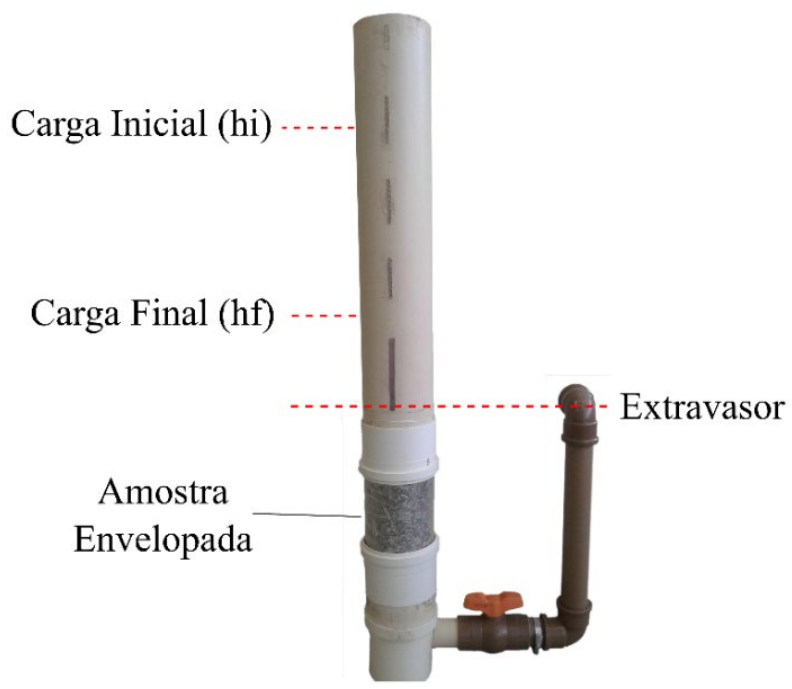

Figura 2. Permeâmetro de carga variável para determinação da condutividade hidráulica

A determinação da condutividade hidráulica foi realizada inserindo a amostra de concreto permeável envelopada no permeâmetro e preenchendo a coluna de carga do equipamento com água até um nível inicial (hi). Em seguida foi medido o tempo necessário (t) para a água se deslocar até o nível final (hf). Neste estudo utilizou-se uma carga inicial de $50 \mathrm{~cm}$ e carga final de $10 \mathrm{~cm}$, ambos medidos em relação ao nível do extravasor. A condutividade hidráulica pode ser determinada pela equação 3, desenvolvida a partir da Lei de Darcy.

$$
\mathrm{k}=\frac{\mathrm{aL}}{\mathrm{At}} \ln \left(\frac{\mathrm{h}_{\mathrm{i}}}{\mathrm{h}_{\mathrm{f}}}\right)
$$

Em que $\mathrm{k}$ é a condutividade hidráulica $(\mathrm{cm} / \mathrm{s})$, a é a área da seção transversal da amostra $\left(\mathrm{cm}^{2}\right)$, A é a área da seção transversal da coluna de carga $\left(\mathrm{cm}^{2}\right)$, L é a altura da amostra $(\mathrm{cm})$, t é o tempo necessário (s) para o nível d'água se deslocar do nível inicial hi $(\mathrm{cm})$ para o nível final hf $(\mathrm{cm})$. 


\section{RESULTADOS}

As 12 (doze) misturas de concreto permeável utilizadas neste estudo apresentaram porosidades no estado fresco variando de 15 a $30 \%$, permitindo uma análise dentro da faixa usual reportada na literatura (Zhong e Wille, 2015; Ibrahim et al., 2014). As porosidades no estado fresco e endurecido foram determinadas e a relação entre estas descritas a seguir.

\subsection{Porosidade no Estado Fresco}

Os resultados de porosidade no estado fresco determinados para as misturas de concreto $\mathrm{g} / \mathrm{c}=3,10$ e $\mathrm{g} / \mathrm{c}=4,68$ submetidos aos diferentes processos de compactação estão ilustrados na Figura $3 . \mathrm{Na}$ figura, as colunas de um mesmo grupo seguidas de mesma letra não diferem significativamente no Teste de Tukey a um nível de significância de 5\%.

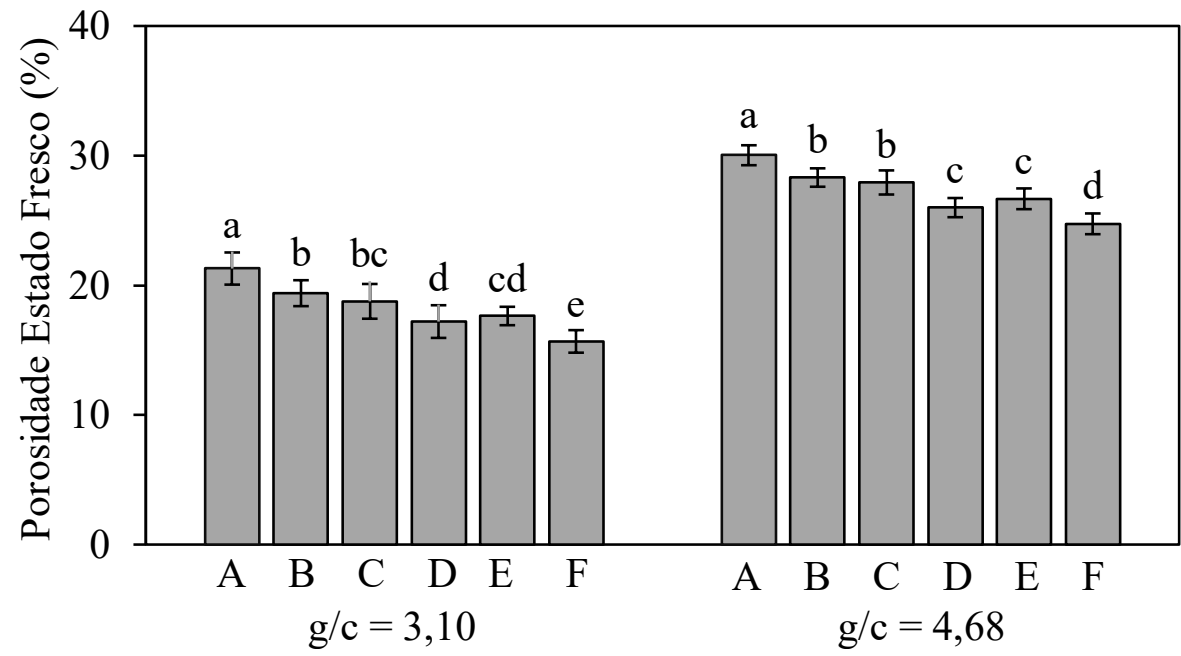

\begin{tabular}{|c|c|c|}
\hline 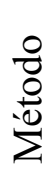 & 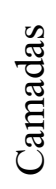 & $\begin{array}{l}\mathscr{d} \\
\frac{0}{0} \\
0 \\
\text { Z }\end{array}$ \\
\hline A & 2 & 10 \\
\hline B & 3 & 6 \\
\hline $\mathrm{C}$ & 2 & 15 \\
\hline D & 3 & 10 \\
\hline $\mathrm{E}$ & 2 & 20 \\
\hline $\mathrm{F}$ & 3 & 14 \\
\hline
\end{tabular}

Figura 3. Porosidade no estado fresco das misturas $\mathrm{g} / \mathrm{c}=3,10 \mathrm{e} \mathrm{g} / \mathrm{c}=4,68$.

Na Figura 3 é possível constatar que cada uma das misturas foi influenciada significativamente pelo esforço de compactação (número de golpes) aplicado. Os resultados demonstram que embora a proporção agregado/cimento tenha se mantido constante em cada grupo, a porosidade obtida em cada concreto permeável variou com o aumento da compactação. No grupo $\mathrm{g} / \mathrm{c}=3,10$ a variação da compactação promoveu uma redução de porosidade de até 5,6\%, enquanto no grupo $\mathrm{g} / \mathrm{c}=4,68$ essa variação foi de até $5,3 \%$. Por outro lado, a variação da relação $\mathrm{g} / \mathrm{c}$ para uma mesma compactação promoveu uma variação média de $9,0 \%$ na porosidade. Isso pode ser explicado pela diferença no volume de pasta de cimento entre as misturas. Os concretos permeáveis do grupo $\mathrm{g} / \mathrm{c}=3,10$ apresentaram um maior volume de pasta de cimento quando comparados ao grupo $\mathrm{g} / \mathrm{c}=4,68$. Esse volume adicional de pasta passa a ocupar os vazios do agregado graúdo, reduzindo a porosidade do concreto (Torres et al., 2015). Os resultados expostos na Figura 3 demonstram que no processo de dosagem de concretos permeáveis a definição do traço do concreto (relação g/c) não é suficiente para controlar a porosidade e as demais propriedades do material. O controle do processo de compactação também é fundamental na produção de pavimentos permeáveis, uma vez que afeta diretamente a porosidade (Suleiman et al., 2006; Bonicelli et al., 2013).

$\mathrm{O}$ concreto permeável do grupo $\mathrm{g} / \mathrm{c}=3,10$ compactado pelo método $\mathrm{F}$ apresentou uma porosidade de $15,67 \%$, que é uma região próxima ao limite inferior sugerido na literatura. Uma compactação mais energética causaria uma redução excessiva da porosidade, comprometendo o desempenho hidráulico do material. Como consequência o pavimento não apresentaria uma capacidade de 
infiltração de águas pluviais suficiente, principalmente a longo prazo pela ocorrência da colmatação dos poros.

\subsection{Porosidade no Estado Endurecido e Porosidade Aberta}

Os resultados de porosidade no estado endurecido e de porosidade aberta estão representados nas Figuras 4 e 5, respectivamente. Nas figuras as colunas de um mesmo grupo seguidas de mesma letra não diferem significativamente no Teste de Tukey a um nível de significância de 5\%.

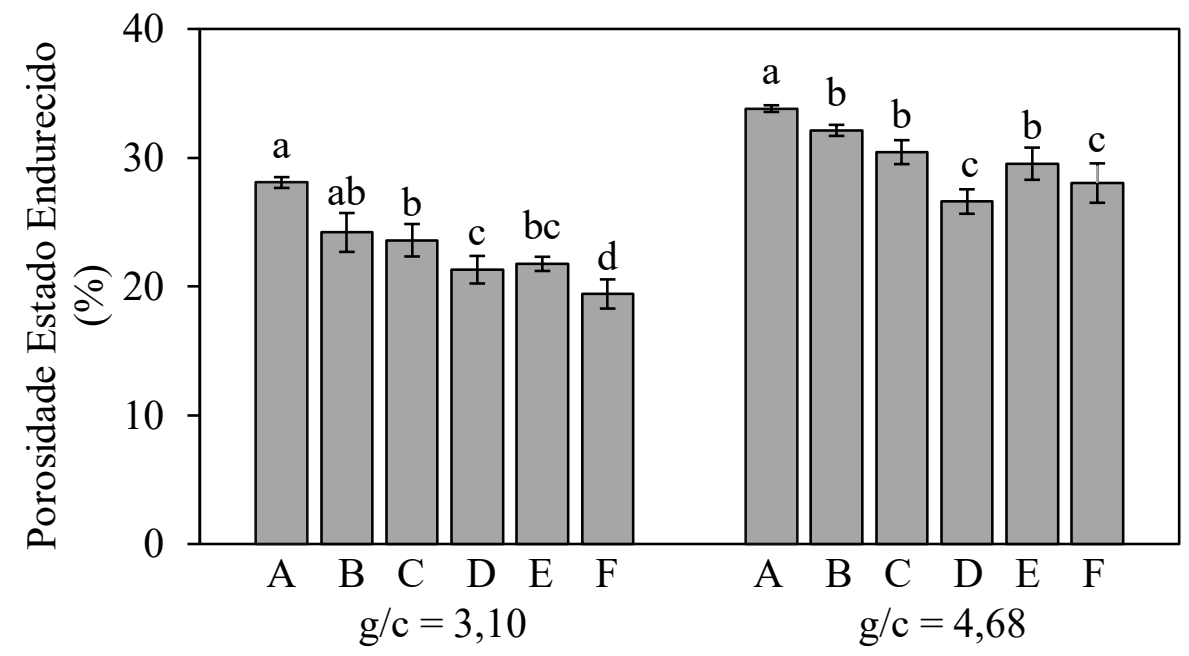

\begin{tabular}{|c|c|c|}
\hline $\begin{array}{l}\frac{0}{0} \\
\text { 过 } \\
\sum\end{array}$ & 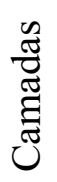 & 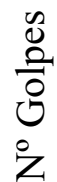 \\
\hline $\mathrm{A}$ & 2 & 10 \\
\hline B & 3 & 6 \\
\hline $\mathrm{C}$ & 2 & 15 \\
\hline D & 3 & 10 \\
\hline $\mathrm{E}$ & 2 & 20 \\
\hline $\mathrm{F}$ & 3 & 14 \\
\hline
\end{tabular}

Figura 4. Porosidade no estado enfurecido das misturas $\mathrm{g} / \mathrm{c}=3,10$ e $\mathrm{g} / \mathrm{c}=4,68$

É possível notar na Figura 4 que a porosidade total, determinada pela metodologia ASTM C1754M (ASTM, 2012), foi superior à porosidade no estado fresco. No entanto observa-se que o efeito do método de compactação foi coerente com o observado no estado fresco. Analisando a Figura 5 é possível notar que a porosidade aberta foi inferior à porosidade no estado endurecido.

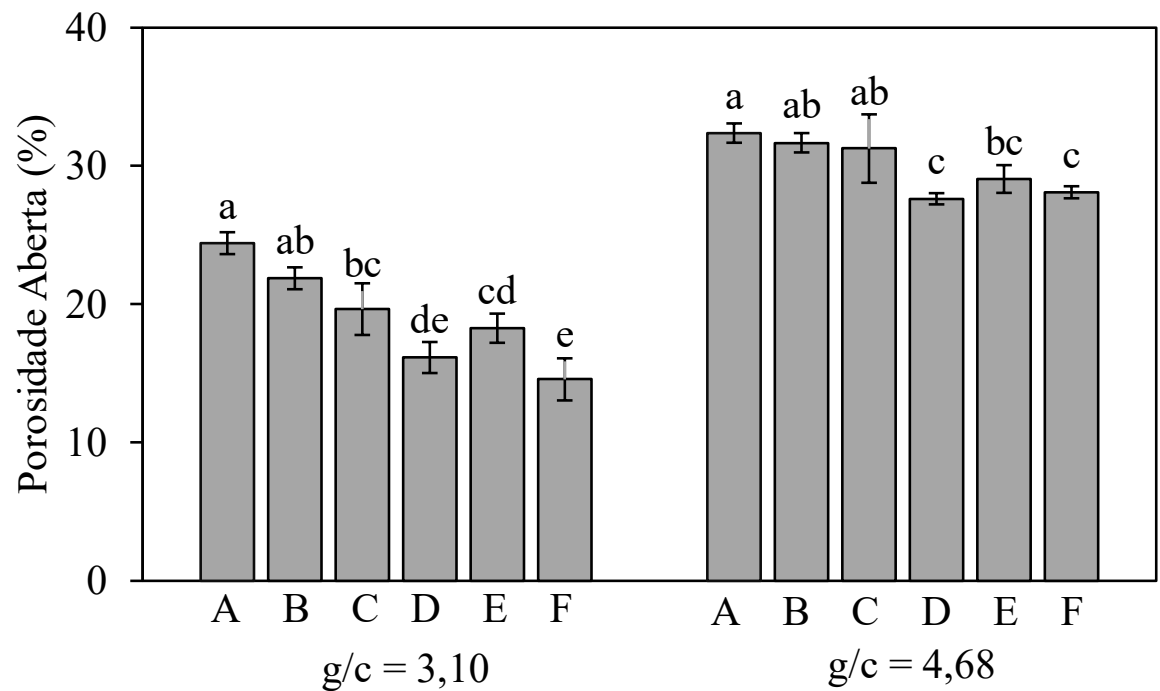

\begin{tabular}{|c|c|c|}
\hline 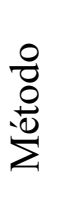 & 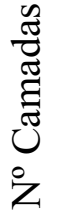 & $\begin{array}{l}\tilde{0} \\
\frac{0}{0} \\
0 \\
0 \\
\text { Z }\end{array}$ \\
\hline A & 2 & 10 \\
\hline B & 3 & 6 \\
\hline $\mathrm{C}$ & 2 & 15 \\
\hline D & 3 & 10 \\
\hline $\mathrm{E}$ & 2 & 20 \\
\hline $\mathrm{F}$ & 3 & 14 \\
\hline
\end{tabular}

Figura 5. Porosidade aberta das misturas $\mathrm{g} / \mathrm{c}=3,10 \mathrm{e} \mathrm{g} / \mathrm{c}=4,68$

Essa variação pode ser atribuída à presença de poros fechados, os quais não são acessíveis à água. Nos concretos do grupo $\mathrm{g} / \mathrm{c}=3,10$ essa diferença foi em média 3,9\%, enquanto nas misturas $\mathrm{g} / \mathrm{c}=4,68$ a diferença foi inferior a $1,0 \%$. Isso demonstra que os concretos com $\mathrm{g} / \mathrm{c}=3,10$ apresentaram uma maior quantidade de poros fechados em relação ao segundo grupo. Isso se deve 
ao maior volume de pasta de cimento na mistura, que além de reduzir a porosidade total pode reduzir a conectividade dos poros, formando vazios isolados (Montes et al., 2005; Torres et al., 2015). Nos métodos de compactação mais energéticos (D, E e F) o volume de poros fechados foi superior. Isso demonstra que a compactação afeta não apenas a porosidade global do concreto permeável, mas também a conectividade dos poros. Embora isso possa limitar a permeabilidade, uma energia de compactação mínima é necessária para conferir aos concretos resistência nos pontos de contato entre agregados e evitar manifestações patológicas como a desagregação dos pavimentos (Adil et al., 2020).

\subsection{Relação entre as Porosidades no Estado Fresco e no Estado Endurecido}

Comparando-se as porosidades obtidas por meio de cada metodologia, é possível notar que a porosidade no estado endurecido foi superior à porosidade no estado fresco em todos os concretos. Isto pode ser observado na Figura 6a, que representa a relação entre ambas as porosidades de cada amostra analisada.
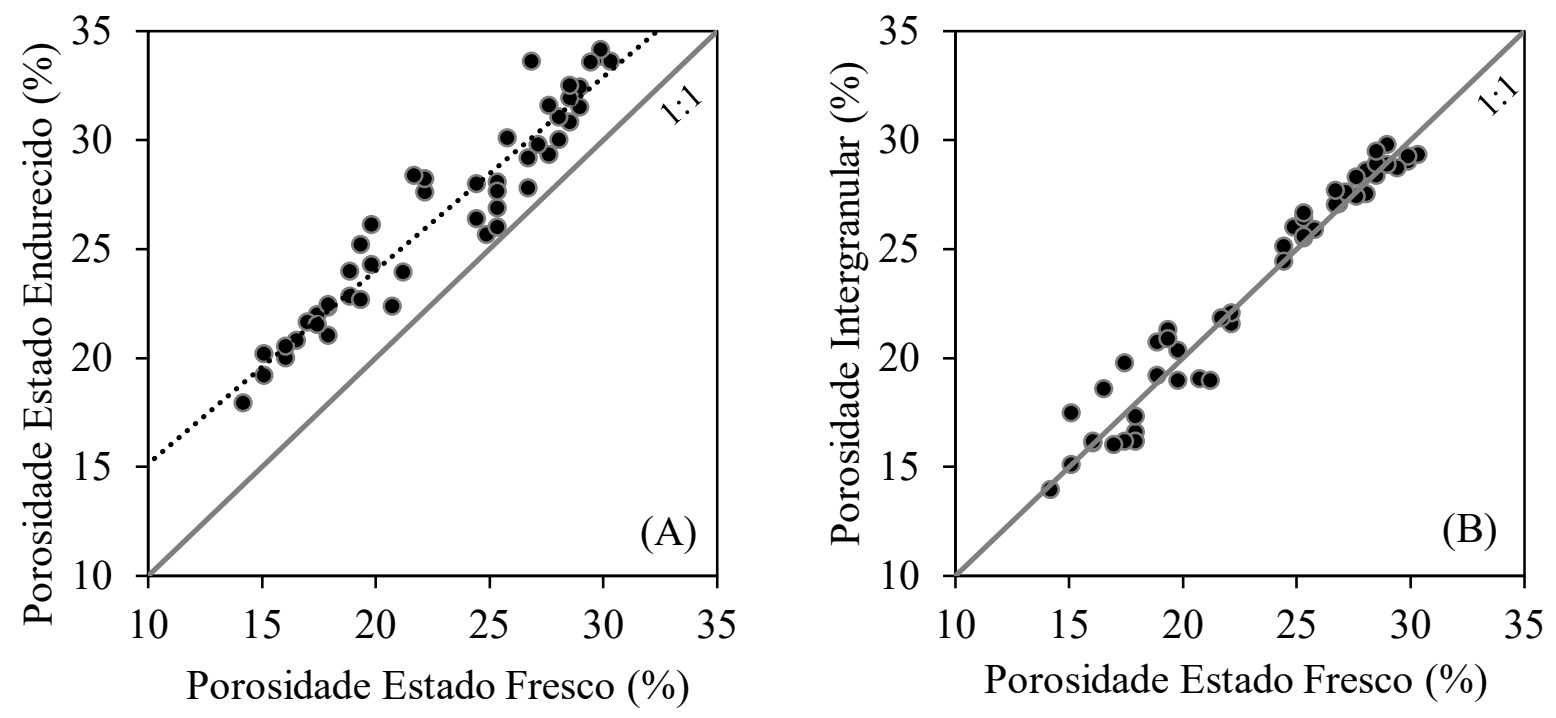

Figura 6. (a) Relação entre a porosidade no estado fresco e no estado endurecido e (b) relação entre a porosidade no estado fresco e intergranular

Na figura é possível verificar que a linha de tendência se encontra deslocada da reta de igualdade entre as porosidades. A variação entre a porosidade no estado fresco e estado endurecido foi mais acentuada nos concretos com porosidade inferior a 20\%, decrescendo nas amostras mais porosas. Essa diferença se deve ao fato de que cada metodologia de caracterização considera diferentes tipos de poros no cálculo da porosidade. No estado fresco a metodologia descrita na ASTM C1688M (ASTM, 2014) utiliza a densidade teórica do concreto para realizar a estimativa de porosidade. Neste momento, a pasta de cimento é considerada como sendo isenta de poros. Desta forma, essa técnica de caracterização resulta na porosidade intergranular do concreto permeável. Por outro lado, no estado endurecido a ASTM C1754M (ASTM, 2012) estabelece que a porosidade seja determinada pelo procedimento de pesagem hidrostática após um período de imersão em água de 30 minutos. Nesta condição, não apenas os poros intergranulares serão saturados, mas também os vazios da pasta de cimento. Portanto, a utilização dessa metodologia permite o cálculo da porosidade total do concreto permeável. Pode-se concluir que a diferença entre a porosidade no estado endurecido e no estado fresco é equivalente ao volume de vazios da pasta de cimento. 
Por meio do levantamento da densidade aparente do fragmento de concreto permeável, foi possível calcular a porosidade intergranular do concreto permeável, conforme a equação 2. Os resultados obtidos foram comparados com a porosidade no estado fresco, conforme apresentado na Figura $6 \mathrm{~b}$. Observando a Figura 6 b é possível verificar uma correspondência entre a porosidade no estado fresco e a porosidade intergranular calculada, uma vez que os dados se aproximam da reta de igualdade. Desta forma, é possível considerar a porosidade no estado fresco como uma forma de avaliar os poros intergranulares do concreto permeável. Por outro lado, a porosidade no estado endurecido também considera os poros da matriz cimentícia, sendo uma forma de caracterizar a porosidade total.

\subsection{Relação entre Porosidade e Condutividade Hidráulica}

Conforme discutido, cada metodologia de caracterização do concreto permeável pode resultar em um tipo de porosidade distinta. Embora em muitos estudos não seja feita nenhuma distinção, é fundamental conhecer cada tipo de porosidade e o seu impacto nas demais propriedades do material. Uma das propriedades do concreto permeável afetada diretamente pela porosidade é a condutividade hidráulica $(\mathrm{k})$. Com o intuito de avaliar o tipo de porosidade que apresenta maior impacto nessa propriedade, foram realizados ajustes de regressão não-linear baseados no modelo de Kozeny-Carman (Chandrappa e Biligiri, 2016; Debnath e Sarkar, 2019), conforme a equação (4).

$$
\mathrm{k}=\mathrm{A} \cdot \frac{\mathrm{P}^{3}}{(100-\mathrm{P})^{2}}
$$

$\mathrm{Na}$ equação 4, a condutividade hidráulica (k) é estimada por meio da porosidade do material (P) e de um coeficiente A que depende das características da rede de poros, como tortuosidade, conectividade e dimensão (Neithalath et al., 2010; Zhong et al., 2016). O modelo de KozenyCarman foi ajustado utilizando-se a porosidade intergranular, porosidade aberta e porosidade total como estimadores da condutividade hidráulica, conforme apresentado nas figuras Figura 7 e 8.
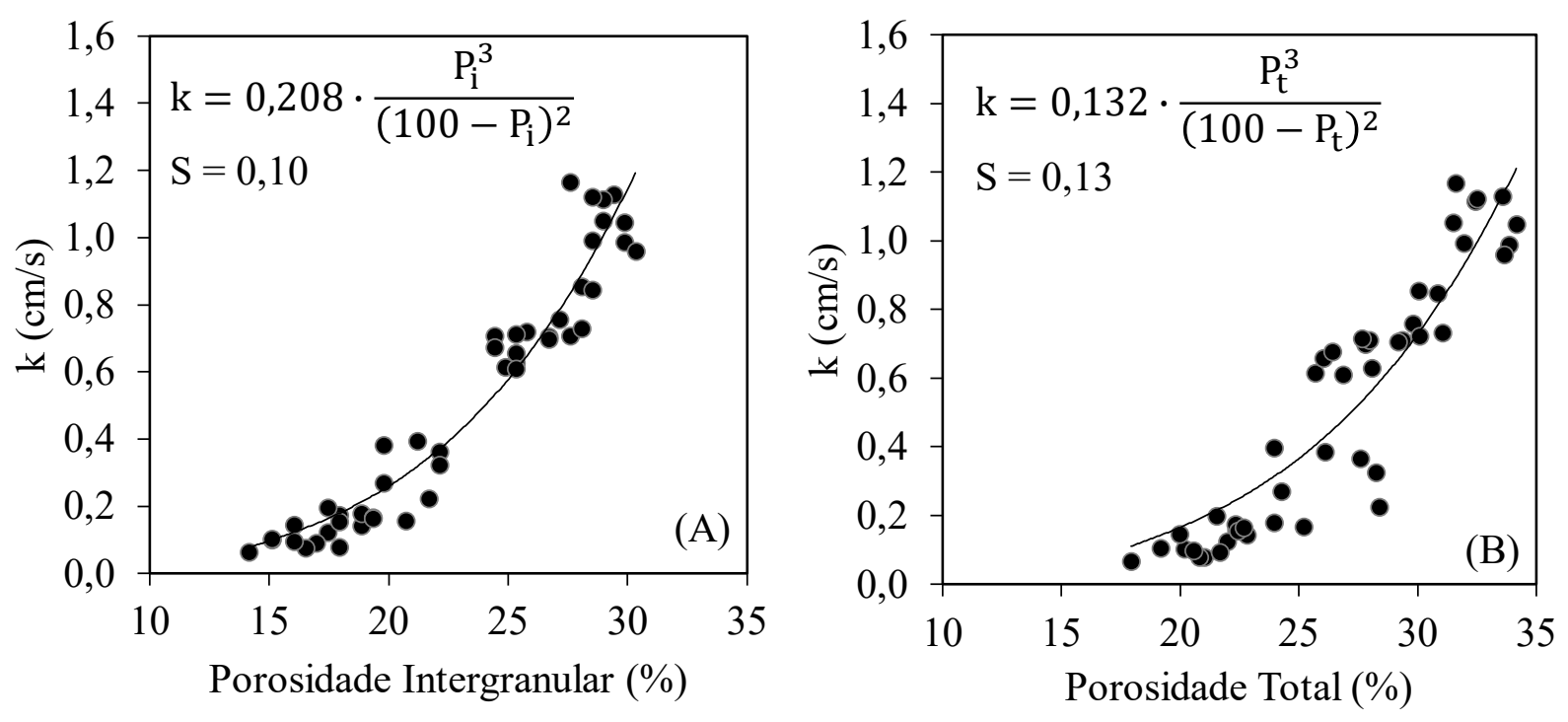

Figura 7. Modelo de Kozeny-Carman para previsão de permeabilidade utilizando (a) a porosidade intergranular como estimador e (b) a porosidade total como estimador

Na Figura 7a verifica-se que utilizando a porosidade intergranular como preditor houve um bom ajuste dos dados experimentais ao modelo proposto, constatando-se um erro padrão de $0,10 \mathrm{~cm} / \mathrm{s}$. 
Por outro lado, quando a porosidade total foi utilizada no modelo de regressão (Figura $7 \mathrm{~b}$ ) houve uma maior dispersão dos resultados experimentais, evidenciado pelo aumento no erro padrão para $0,13 \mathrm{~cm} / \mathrm{s}$. O maior erro neste modelo pode ser justificado pelo fato de que os poros da matriz cimentícia (considerados na porosidade total) não contribuírem diretamente para a condução de água no interior do concreto.

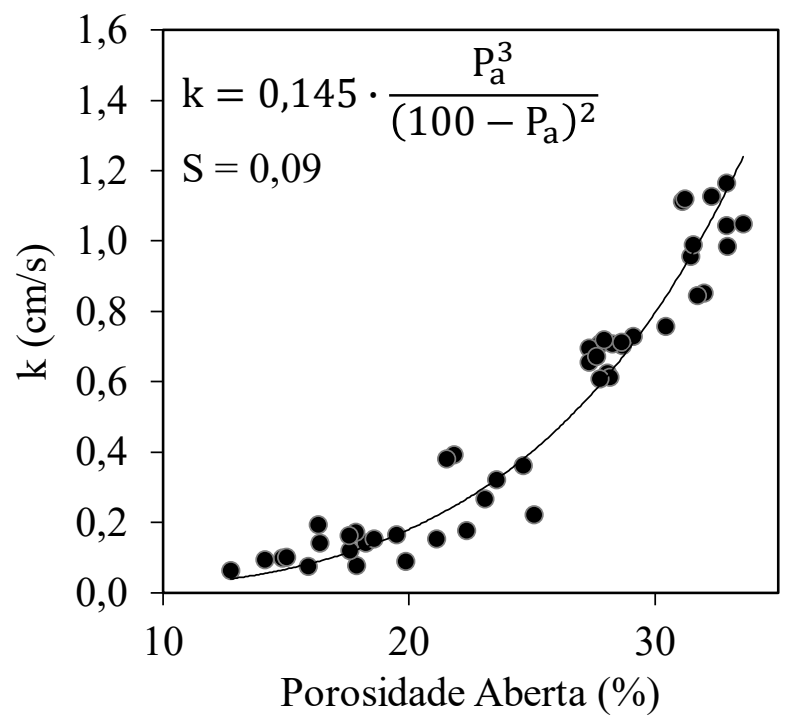

Figura 8. Modelo de Kozeny-Carman para previsão de permeabilidade utilizando a porosidade aberta como estimador

Na Figura 8 observa-se que a porosidade aberta foi o melhor parâmetro para descrever o comportamento da condutividade hidráulica do concreto permeável, com um erro padrão de 0,09 $\mathrm{cm} / \mathrm{s}$. O melhor desempenho da porosidade aberta como parâmetro de estimação da permeabilidade pode ser atribuído ao fato de que apenas os poros conectados, e que participam efetivamente na condução de água, são considerados. Na Figura 9 estão ilustrados os dados experimentais deste estudo comparados aos resultados descritos na literatura.

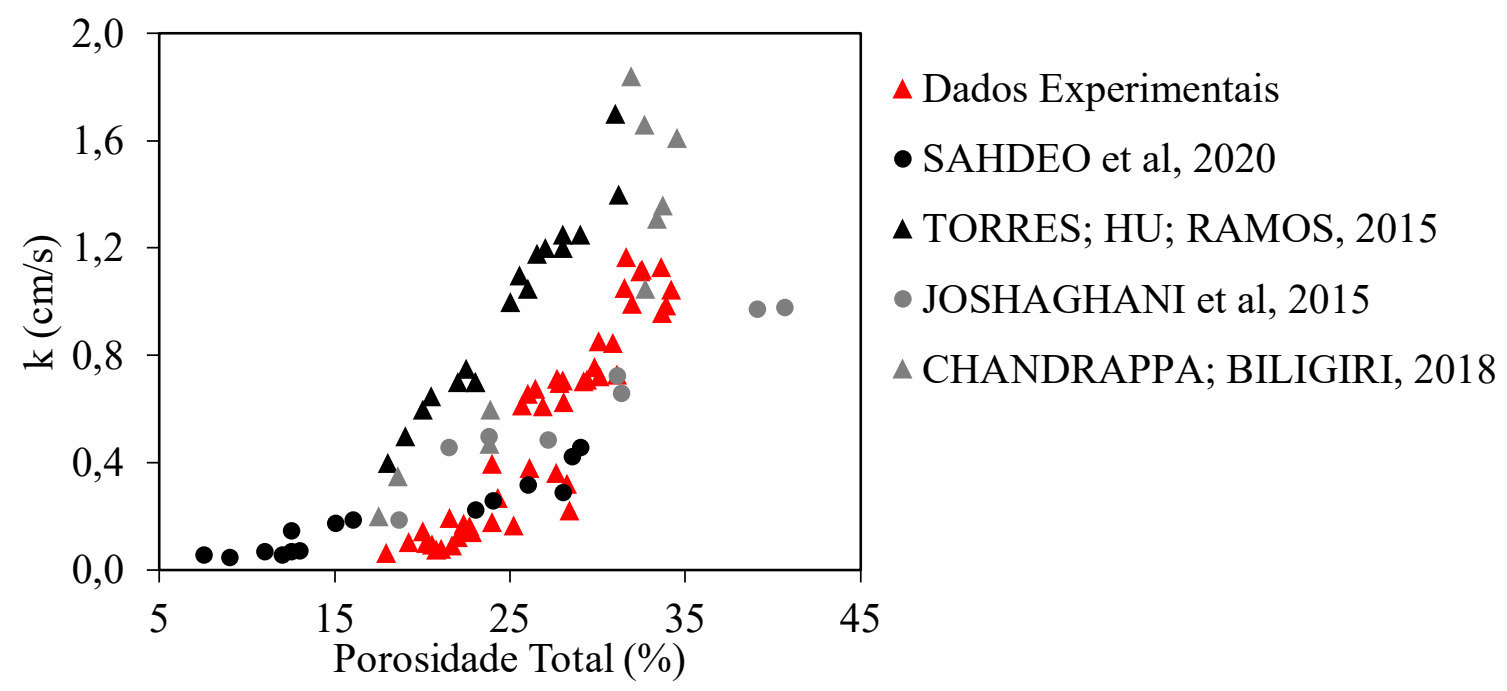

Figura 9. Comparação dos resultados de porosidade e condutividade hidráulica com dados experimentais da literatura 
Conforme a Figura 9, os resultados de condutividade hidráulica foram coerentes com os valores usuais relatados na literatura. Observa-se uma tendência similar em todos os estudos, com crescimento exponencial da condutividade hidráulica com o aumento da porosidade. A maioria dos dados se concentrou entre 15 e $35 \%$ de porosidade, em concordância com a faixa usual exposta na literatura (Zhong e Wille, 2015).

A condutividade hidráulica é uma das principais propriedades no desenvolvimento de pavimentos drenantes, uma vez que estes devem apresentar uma capacidade de infiltração de água compatível com a precipitação de projeto. Modelos hidráulicos permitem a definição de uma porosidade que resulte em uma condutividade hidráulica suficiente para um desempenho adequado dos pavimentos porosos. Além disso, é possível estabelecer coeficientes de permeabilidade com uma margem de segurança, prevendo-se a ocorrência da colmatação dos poros. Isto permite a prolongação da vida útil do pavimento, garantindo a sua eficiência sem a necessidade de manutenções constantes.

Dessa forma, a estimação da condutividade hidráulica é de grande importância na dosagem de misturas de concreto permeável, sendo o controle da porosidade uma forma de garantir a qualidade e o desempenho do pavimento drenante. Embora o comportamento hidráulico do concreto dependa de outros fatores referentes as características dos poros, a porosidade aberta é um parâmetro com potencial aplicação prática na dosagem de misturas de concreto permeável.

\subsection{Relação entre Porosidade e Resistência à Compressão}

A resistência mecânica do concreto permeável também é afetada pela sua porosidade, porém de forma inversamente proporcional. Assim, ainda que o aumento da porosidade seja benéfico para aprimorar a capacidade hidráulica do material, a resistência mecânica pode ser insuficiente para a aplicação em pavimentos. Dessa forma, com o objetivo de analisar a influência dos tipos de poros no desempenho mecânico do concreto, foram elaborados modelos de regressão exponenciais. As porosidades intergranular, aberta e total foram utilizadas como preditores de resistência à compressão, conforme ilustrado nas Figuras 10 e 11.
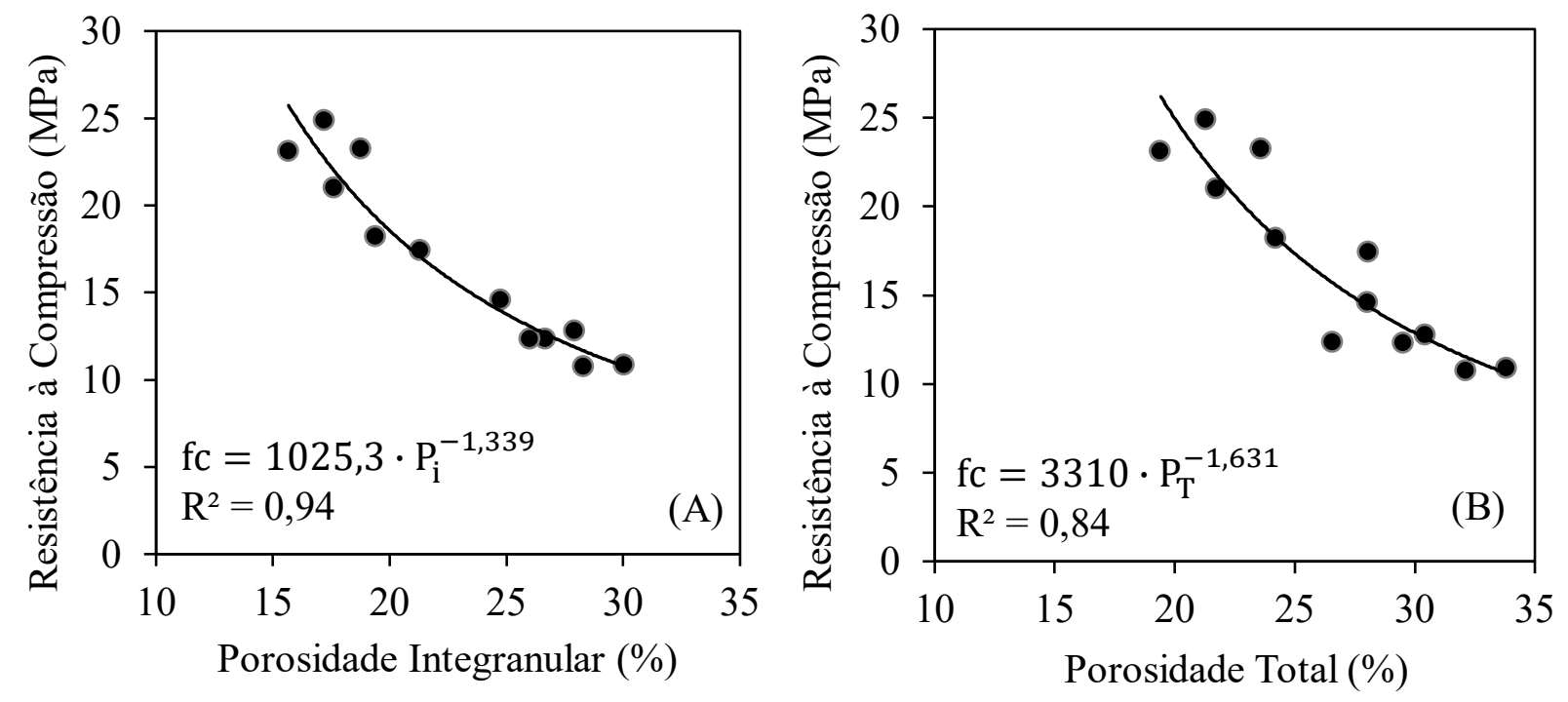

Figura 10. Modelos de regressão de resistência à compressão utilizando (a) a porosidade intergranular como estimador e (b) a porosidade total como estimador 
Na Figura 10a é possível constatar uma forte correlação $\left(\mathrm{R}^{2}=0,94\right)$ entre a porosidade intergranular e a resistência à compressão. Embora a porosidade total considere os poros da pasta de cimento, que influenciam a resistência mecânica da matriz cimentícia, o modelo utilizando esse parâmetro como preditor não apresentou melhor coeficiente de determinação $\left(\mathrm{R}^{2}=0,84\right)$. Neste caso, como todos os concretos foram confeccionados com mesma relação água/cimento, é esperado que a matriz cimentícia apresente comportamento mecânico similar. Desta forma, a resistência mecânica do concreto permeável foi influenciada principalmente pela porosidade intergranular.

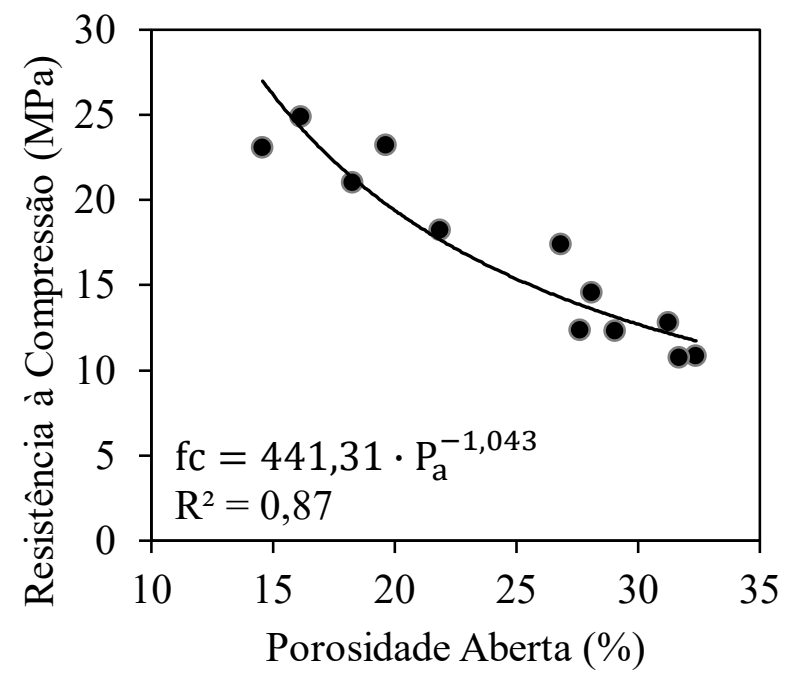

Figura 11. Modelos de regressão de resistência à compressão em função da porosidade aberta

O modelo utilizando a porosidade aberta como preditor da resistência mecânica (Figura 11) também apresentou desempenho inferior $\left(\mathrm{R}^{2}=0,87\right)$ quando comparado à porosidade intergranular. Isso pode ser justificado pelo modelo não considerar os poros fechados, prejudicando assim a sua capacidade em explicar a variação da resistência à compressão do concreto permeável. Portanto, a porosidade intergranular pode ser uma opção na dosagem de concretos permeáveis, visto que essa variável permite estabelecer uma relação com a resistência mecânica do material. $\mathrm{Na}$ Figura 12 estão representados os dados experimentais em comparação com valores da literatura.

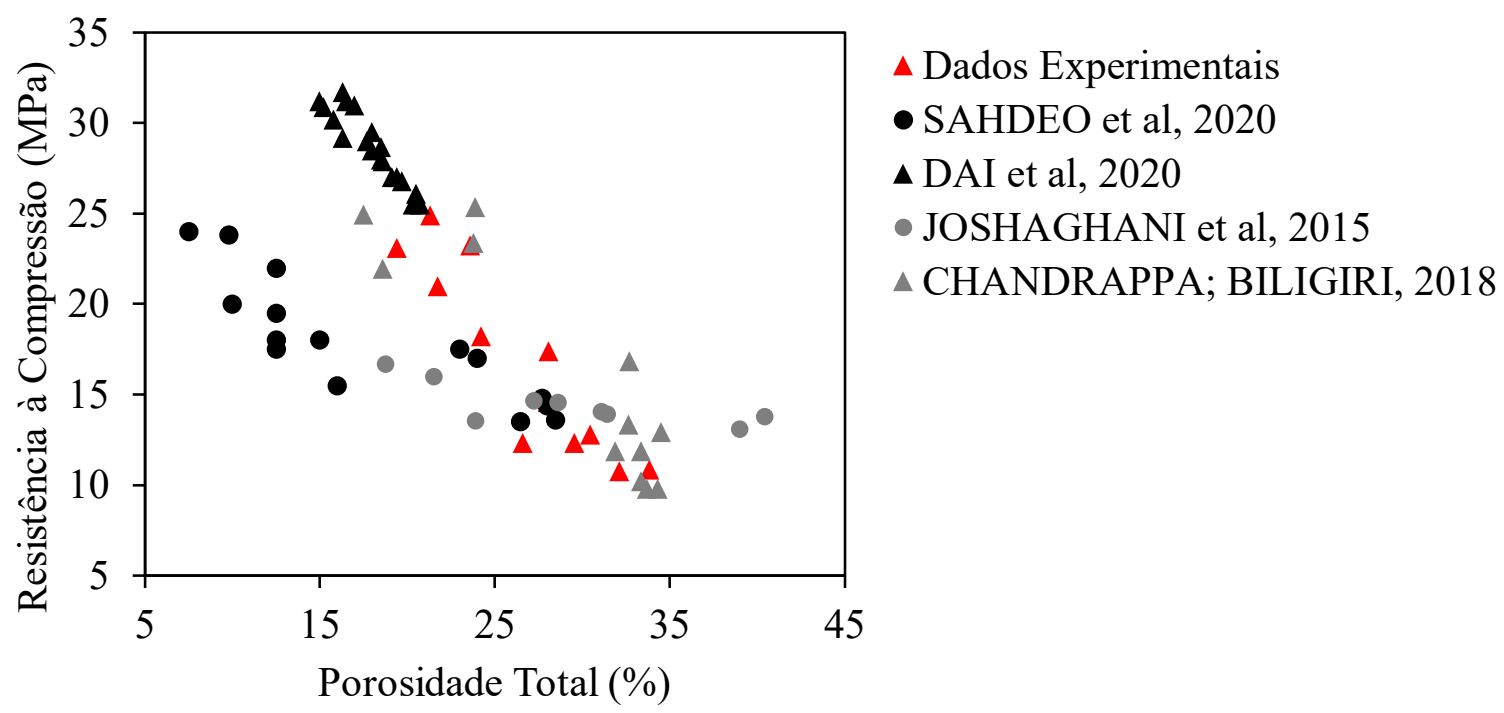

Figura 12. Comparação dos resultados de porosidade e resistência com dados valores da literatura 
Na Figura 12 observa-se que os resultados de resistência à compressão obtidos neste estudo variaram de forma similar aos dados dos estudos selecionados. Projetando-se porosidades de 15 a $35 \%$ é possível ajustar a resistência à compressão do concreto permeável em uma faixa de 10 a 30 $\mathrm{MPa}$. Embora concretos com porosidade superior a 30\% apresentem um desempenho hidráulico superior (Figura 9), a resistência mecânica geralmente é um fator limitante na dosagem. Por outro lado, porosidades inferiores a $15 \%$ podem oferecer um melhor desempenho mecânico, porém com condutividade hidráulica limitada.

Portanto, a previsão do comportamento mecânico do concreto permeável também é fundamental para garantir a eficiência e durabilidade de pavimentos. A definição da resistência mecânica do concreto permeável deve ser feita com o objetivo de suportar o tráfego de veículos projetado para o pavimento. Um desempenho mecânico insuficiente poderá ocasionar deteriorações, como a ruptura ou a desagregação do material. Dessa forma, o controle da porosidade do concreto permeável possibilita a seleção de uma resistência mecânica em conjunto com uma condutividade hidráulica compatível para a aplicação em pavimentos drenantes.

\section{CONCLUSÕES}

Este estudo abordou a análise de diferentes técnicas de caracterização da porosidade, bem como a sua influência nas propriedades do concreto permeável. Os resultados obtidos demonstraram que a porosidade no estado fresco considera principalmente os poros intergranulares do material. No estado endurecido a caracterização resulta na porosidade total do concreto, uma vez que além dos poros intergranulares os vazios da matriz cimentícia também são considerados. Também foi possível caracterizar a porosidade aberta, excluindo-se os poros isolados do material. Foi possível constatar a importância em se diferenciar cada porosidade no concreto permeável, uma vez que cada propriedade é influenciada por um tipo principal de poro. A permeabilidade se demonstrou como dependente da porosidade aberta do concreto permeável. Esse resultado foi coerente com a literatura, visto que os poros isolados e os vazios da matriz cimentícia não contribuem diretamente na condução de água no interior do material. A resistência à compressão, por sua vez, pôde ser representada com melhor eficiência pela porosidade intergranular. Embora a porosidade aberta seja mais adequada para a previsão do comportamento hidráulico, essa é uma propriedade de difícil previsão, uma vez que depende das características do agregado e do processo de compactação empregado. No entanto, a porosidade intergranular, caracterizada no estado fresco, apresentou boa correlação tanto com as características hidráulicas como mecânicas do concreto permeável. A porosidade intergranular pode ainda ser ajustada pela relação g/c e pelo método de compactação. Assim, a utilização desse parâmetro é útil no processo de dosagem do material, sendo uma forma de estimar as propriedades relevantes para o desempenho adequado de pavimentos drenantes. Dessa forma, o controle da porosidade possibilita o desenvolvimento de misturas de concreto permeável com maior qualidade, visando assim a durabilidade dos pavimentos permeáveis.

\section{AGRADECIMENTOS}

Os autores agradecem ao Laboratório de Materiais de Construção Civil da Universidade Estadual de Ponta Grossa pelo apoio e infraestrutura disponibilizados para o desenvolvimento desta pesquisa.

\section{REFERÊNCIAS}

ACI American Concrete Institute. (2010), ACI 522R-10 Report on pervious concrete. 
Adil, G. et al. (2020), Influence of silica fume on mechanical and durability of pervious concrete. Construction and Building Materials. 247:118453.

Associação Brasileira de Normas Técnicas. (2009), NBR NM 53: Agregado graúdo - Determinação de massa especifica, massa específica aparente e absorção de água. Rio de Janeiro.

ASTM International. (2014), ASTM C1688/C1688M-14a Standard Test Method for Density and Void Content of Freshly Mixed Pervious Concrete.

ASTM International. (2012), ASTM C1754/C1754M-12 Standard Test Method for Density and Void Content of Hardened Pervious Concrete.

Bonicelli, A. et al. (2013), Laboratory analysis for investigating the impact of compaction on the properties of pervious concrete mixtures for road pavements. Advanced Materials Research. 723:409-419.

Chandrappa, A. K., Biligiri, K. P. (2016), Pervious concrete as a sustainable pavement material Research finding and future prospects: A state-of-the-art review. Construction and Building Materials. 111:262-274.

Chandrappa, A. K., Biligiri, K. P. (2016), Comprehensive investigation of permeability characteristics of pervious concrete: A hydrodynamic approach. Construction and Building Materials. 123:627-637.

Chandrappa, A. K., Biligiri, K. P. (2018), Methodology to Develop Pervious Concrete Mixtures for Target Properties Emphasizing the Selection of Mixture Variables. Journal of Transportation Engineering, Part B: Pavements. 144(3): 04018031.

Dai, Z. (2020), Multi-modified effects of varying admixtures on the mechanical properties of pervious concrete based on optimum design of gradation and cement-aggregate ratio. Construction and Building Materials. 233: 117178.

Debnath, B.; Sarkar, P. P. (2019), Permeability prediction and pore structure feature of pervious concrete using brick as aggregate. Construction and Building Materials. 213:643-651.

Deo, O., Neithalath, N. (2010), Compressive behavior of pervious concretes and a quantification of the influence of random pore structure features. Materials Science and Engineering A. 528:402412.

Gaedicke, C. et al. (2015), Effect of recycled materials and compaction methods on the mechanical properties and solar reflectance index of pervious concrete. Revista Ingeniería de Construcción. 30(3):159-167.

Ibrahim, A. et al. (2014), Experimental study on Portland cement pervious concrete mechanical and hydrological properties. Construction and Building Materials. 50:524-9.

Joshaghani, A. et al. (2015), Optimizing pervious concrete pavement mixture design by using the Taguchi method. Construction and Building Materials. 101:317-325. 
Kia A. et al. (2017), Clogging in permeable concrete: A review. Journal of Environmental Management. 193:221-223.

Mehta, P. K., Monteiro, P. J. M. “Concreto: Microestrutura, Propriedades e Materiais”. IBRACON, São Paulo, Brasil, p. 782.

Montes, F. et al. (2005), A new test method for porosity measurements of Portland cement pervious concrete. Journal of ASTM International. 2(1).

Neithalath, N. et al. (2010), Characterizing pore volume, sizes, and connectivity in pervious concretes for permeability prediction. Materials Characterization. 61(8):802-813.

Rao, Y. et al. (2020) Vertical distribution of pore-aggregate-cement paste in statically compacted pervious concrete. Construction and Building Materials. 237:117605.

Sahdeo, S. K. et al. (2020) Effect of mix proportion on the structural and functional properties of pervious concrete paving mixtures. Construction and Building Materials. 255: 119260.

Suleiman, M. T. et al. (2006) "Effect of compaction energy on pervious concrete properties". In: Proceedings of the 2006 NRMCA Concrete Technology Forum - Focus on Pervious Concrete, NRMCA, Nashville.

Torres, A., Hu, J. (2016), An alternate test method for the void contente of pervious concrete. Advances in Civil Engineering Materials. 5(1):24-45.

Torres, A. et al. (2015), The effect of the cementitious paste thickness on the performance of pervious concrete. Construction and Building Materials. 95:850-859.

Xie, X. et al. (2018), Maximum paste coating thickness without voids clogging of pervious concrete and its relationship to the rheological properties of cement paste. Construction and Building Materials, 168: 732-746.

Zhong, R., Wille, K. (2015), Material design and characterization of high performance pervious concrete. Construction and Building Materials, 98:51-60. 